\title{
Ubiquitin-specific protease 22 is associated with poor prognosis in neuroblastoma
}

\author{
Qu Zhibo ${ }^{1,2, A-D}$, Liu Lianxin ${ }^{1, A, F}$ \\ 1 Department of Heptic Surgery, First Affiliated Hospital of Harbin Medical University, China \\ ${ }^{2}$ Department of General Surgery, Harbin Children's Hospital, China \\ A - research concept and design; $\mathrm{B}$ - collection and/or assembly of data; $\mathrm{C}$ - data analysis and interpretation; \\ $D$ - writing the article; $E$ - critical revision of the article; $F$ - final approval of the article
}

\section{Address for correspondence \\ Liu Lanxin \\ E-mail:liu1970@ems.hrbmu \\ Funding sources \\ This study was supported by the Foundation of Harbin Science and Technology Bureau (grant No. 2014RFQGJ124).}

\section{Conflict of interest}

None declared

Received on August 13, 2018

Reviewed on September 30, 2018

Accepted on December 5, 2019

Published online on March 25, 2020

\begin{abstract}
Background. Ubiquitin-specific protease 22 (USP22) alters histone ubiquitination and is considered to be an oncogenic factor involved in tumor progression. The USP22 aberrance has been implicated in several malignancies, but whether USP22 plays a role in neuroblastoma (NB) remains unclear. To the best of our knowledge, the clinicopathological significance of USP22 expression in NB has not been previously reported in the English-language medical literature.
\end{abstract}

Objectives. The aim of this study was to investigate the role of USP22 and its association with potential targets in patients with NB.

Material and methods. The potential clinicopathological significance of USP22 expression in NB was studied using immunohistochemistry, immunohistochemical staining assessment and statistical analyses.

Results. Based on the immunohistochemical analysis, the USP22 protein was detected more manifestly in NB tissues than in healthy peritumoral tissue. Furthermore, an association between USP22, lymph node metastasis and NB clinical stage was observed, whereby the level of USP22 protein was higher in stage III-IV specimens than in stage $1-\|$ specimens $(p<0.05)$. Furthermore, tumors expressing USP22 were associated with poorer patient prognosis than the USP22-negative tumors. The multivariate Cox regression analysis suggested that the level of USP22 protein is a predictive factor for survival $(p<0.05)$.

Conclusions. Our results indicate a significant association between USP22 level and poor prognosis in NB. Thus, USP22 represents a valuable biomarker for predicting the outcome of patients with NB.

Key words: neuroblastoma, ubiquitin-specific protease 22, poor prognostic

Cite as

Zhibo Q, Lianxin L. Ubiquitin-specific protease 22 is associated with poor prognosis in neuroblastoma. Adv Clin Exp Med. 2020;29(3):295-300. doi:10.17219/acem/115089

DOI

10.17219/acem/115089

\section{Copyright}

Copyright by Author(s)

This is an article distributed under the terms of the

Creative Commons Attribution 3.0 Unported (CC BY 3.0)

(https://creativecommons.org/licenses/by/3.0/) 


\section{Introduction}

Neuroblastoma (NB) is a rare type of cancer that develops in children and infants at an annual incidence rate of between 6 and 10 per million. ${ }^{1}$ Originating in primitive precursors of the sympathetic nervous system, NB is mostly observed in the adrenal medulla and sympathetic nerve ganglia. ${ }^{2}$ In addition, NB is characterized by rapid growth and shows a strong capacity for invasion and metastasis, which often cause the advanced expansion and outward invasion of the tumor into neighboring tissues. Despite substantial progress in the treatment of NB, including surgical techniques and chemotherapy, the 5-year survival rate remains low, especially in patients in clinical stages III and IV. ${ }^{3}$ Thus, identifying new biomarkers for the effective diagnosis of NB and predicting its therapeutic outcome is highly valuable.

Ubiquitin-specific protease 22 (USP22) is an element of the 11-Polycomb/cancer stem cell signature that is involved in the alteration of histone ubiquitination, which makes it a valuable marker for predicting the therapeutic response of individual patients with cancer. ${ }^{4}$ Elevated USP22 expression is related to a poor prognosis in colorectal, gastric, liver, lung, and breast cancers. ${ }^{5-9}$ USP22, as a component of the human Spt-Ada-Gen5-acetyltransferase (hSAGA) complex, is at the core of several physiological and pathological processes and is directly implicated in cell-cycle progression and transcriptional regulation. ${ }^{10}$ As a multi-subunit complex, hSAGA is organized into several functional submodules, including the deubiquitinating module (DUB), the histone acetyltransferase (HAT) module, and the suppressor of Ty (SPT) and TATA-binding protein-associated factor (TAF) modules. The USP22 is the essential protein linked to the DUB module. It modifies histones $\mathrm{H} 2 \mathrm{~A}$ and $\mathrm{H} 2 \mathrm{~B}$, which facilitate a variety of cellular events, including gene regulation. Therefore, upregulation of USP22 expression will lead to abnormal activation of multiple pathways to promote cell survival, while downregulation of USP22 expression can induce cell cycle arrest at the G0/G1 phase in different types of cancer cells. More specifically, USP22 modulates growth and oncogenic transformation by regulating transcription factors, such as BMI-1, c-MYC, p53, TRF1, and SIRT1. ${ }^{11-16}$ These findings provide evidence that USP22 is vital in tumor progression and advancement; thus, it is a valuable target as an NB marker.

Nevertheless, no reports have been published on the clinicopathological consequences of USP22 in NB. Therefore, this study aimed to evaluate USP22 expression in tissues from representative patients with NB using an immunohistochemical analysis, and to determine whether there is any association between USP22 and NB clinicopathology, including prognosis.

\section{Material and methods}

\section{Patients}

A total of 68 specimens were acquired from patients (29 boys and 39 girls) at Harbin Children's Hospital, China, between March 2005 and August 2013. The age of the patients ranged from 36 days to 11 years (median age: 4.2 years). A histological analysis established that 48 patients (70.5\%) had lymph node metastases. The pathological diagnosis indicated that all patients had NB, while none had received any treatment prior to diagnosis. The tumor stages were assessed based on the International Neuroblastoma Staging System (INSS). The pathological data were retrieved from the patients' medical histories, and the follow-up records were acquired by phone interviews and outpatient clinical databases. The survival analysis was performed by postoperatively examining the patients with NB periodically until September 2016 or until death (median follow-up time: 52 months). In the histological analysis, the negative control samples consisted of tissue from the same tissue slides, $1 \mathrm{~cm}$ from the tumors. It was necessary to obtain a broad area because it would otherwise be difficult to find enough controls. Thus, the adjacent tissue from the patients served as the negative control samples. The Ethical Committee of Harbin Children's Hospital approved our retrospective study, and written informed consent was provided for each patient's participation from their parents or legal guardians.

\section{Immunohistochemistry}

The USP22 immunohistochemical analysis was performed based on previously published methods. ${ }^{15}$ Briefly, the tissues were post-fixed with formalin, embedded in paraffin and cut into 4-micrometer sections. Subsequently, the sections were deparaffinized in xylene, rehydrated in ethanol solutions of descending concentration and submerged in ethylenediaminetetraacetic acid (EDTA; pH 8). Antigenicity was retrieved by autoclaving the sections at $121^{\circ} \mathrm{C}$ for $5 \mathrm{~min}$. After quenching the endogenous peroxidase in $3 \% \mathrm{H}_{2} \mathrm{O}_{2}$ for 15 min and washing with phosphatebuffered saline (PBS), the sections were incubated with a primary antibody against USP22 (1:200, ab4812; Abcam, Cambridge, UK) overnight at $4^{\circ} \mathrm{C}$. A 30 -minute peroxidaseconjugated streptavidin incubation and diaminobenzidine incubation were subsequently performed, followed by counterstaining with a commercially available hematoxylin to stain the nuclei (H9627; Sigma-Aldrich, St. Louis, USA).

\section{Immunohistochemical staining assessment}

The immunolabeled sections were evaluated independently under a light microscope (Olympus CX41; Olympus, Tokyo, Japan) by 2 experienced pathologists who were 
blinded to the patients' outcomes. A total of 200 cells from 5 randomly selected areas per section were evaluated to examine the expression of USP22 (a semi-quantitative assessment), and the percentage of USP22-positive cells was subsequently estimated. In general, USP22 was mainly detected in the nuclei. The extent and intensity of the immunolabeling were estimated. The extent of the staining was evaluated on a 5 -point scale, where 0 indicated no positive cells, 1 indicated $\leq 25 \%$ positive cells, 2 indicated $26-50 \%, 3$ indicated $51-75 \%$, and 4 indicated $\geq 76 \%$ positive cells. Similarly, the intensity was scored using a 5-point scale as follows: 0 (no staining), 1 (very weak), 2 (weak), 3 (moderate), and 4 (strong). The final score was determined by combining the proportion and intensity scores, and the results were categorized as follows: 0-1 (negative), 1-3 (low) and $\geq 4$ (high).

\section{Statistical analyses}

We used SPSS v. 12.0 software package (SPSS Inc. Chicago, USA) for all the statistical analyses, with the significance level set at $\mathrm{p}<0.05$. To compare the clinical features according to USP22 expression, $\chi^{2}$ test was used, while the Kaplan-Meier method and a log-rank test were used to measure overall survival.

\section{Results}

\section{Expression of USP22 in NB tissues}

To evaluate the contribution of USP22 to oncogenesis and tumor progression/growth, we examined its expression level in 68 NB specimens from patients between the age of 36 days and 11 years. Based on our results, $61.76 \%$ of the analyzed samples were positive for USP22. Strong USP22 staining was detected mainly within the nuclei, while only weak cytoplasmic staining was observed. Furthermore, no obvious expression of USP22 was detected in the tumor-adjacent tissues (Fig. 1).

\section{Association between USP22 and NB clinical hallmarks}

Table 1 summarizes the comparisons of the results between the USP22 immunoreactivity and NB clinicopathological parameters. Based on these results, positive correlations were observed between the expression of USP22 and lymph node metastasis and the NB clinical stage (both $\mathrm{p}<0.05$ ). In particular, USP22 was more enriched in patients at stages III-IV than stages I-II $(\mathrm{p}<0.05)$. However, the results indicated no significant correlation between USP22 expression and age, sex or tumor size and site. In addition, when the study population was divided into 2 groups by age ( $<2$ years and $\geq 2$ years, instead of 3 years as the group cut-off age), the results also indicated that there was no significant association between USP22 and age.

\section{Expression level of USP22 is correlated with a poor disease prognosis}

We assessed the overall survival rate in our patients based on the USP22 expression level. The median overall survival for all 68 specimens was $57.5 \pm 2.1$ months, while the 5 -year survival rate was $32.5 \% \pm 1.9 \%$. However, the survival rate was $23.6 \% \pm 2.1 \%$ in the patients with

Table 1. Relationship between USP22 expression and the clinicopathological features of NB

\begin{tabular}{|c|c|c|c|c|c|c|c|}
\hline \multirow{2}{*}{ Variables } & \multirow{2}{*}{$\begin{array}{l}\text { Number of patients } \\
\qquad(\mathrm{n}=68)\end{array}$} & \multicolumn{3}{|c|}{ USP22 expression } & \multirow{2}{*}{$\begin{array}{l}\text { Positive rate } \\
(\%)\end{array}$} & \multirow{2}{*}{$x^{2}$} & \multirow{2}{*}{$p$-value } \\
\hline & & high & low & negative & & & \\
\hline $\begin{array}{l}\text { Gender } \\
\text { male } \\
\text { female }\end{array}$ & $\begin{array}{l}29 \\
39\end{array}$ & $\begin{array}{l}12 \\
14\end{array}$ & $\begin{array}{r}5 \\
11\end{array}$ & $\begin{array}{l}12 \\
14\end{array}$ & $\begin{array}{l}58.6 \\
64.1\end{array}$ & 0.38 & $>0.05$ \\
\hline $\begin{array}{l}\text { Age } \\
\geq 3 \\
<3\end{array}$ & $\begin{array}{l}30 \\
38\end{array}$ & $\begin{array}{l}12 \\
14\end{array}$ & $\begin{array}{r}6 \\
10\end{array}$ & $\begin{array}{l}12 \\
14\end{array}$ & $\begin{array}{l}60.0 \\
63.2\end{array}$ & 0.29 & $>0.05$ \\
\hline $\begin{array}{l}\text { Tumor size } \\
\geq 5 \mathrm{~cm} \\
<5 \mathrm{~cm}\end{array}$ & $\begin{array}{l}37 \\
31\end{array}$ & $\begin{array}{l}14 \\
12\end{array}$ & $\begin{array}{l}8 \\
8\end{array}$ & $\begin{array}{l}15 \\
11\end{array}$ & $\begin{array}{l}59.4 \\
64.5\end{array}$ & 0.11 & $>0.05$ \\
\hline $\begin{array}{l}\text { Tumor site } \\
\text { adrenal gland } \\
\text { retroperitoneal } \\
\text { neck } \\
\text { thoracic and mediastinal }\end{array}$ & $\begin{array}{r}35 \\
28 \\
3 \\
2\end{array}$ & $\begin{array}{r}13 \\
11 \\
1 \\
1\end{array}$ & $\begin{array}{l}9 \\
6 \\
1 \\
0\end{array}$ & $\begin{array}{r}13 \\
11 \\
1 \\
1\end{array}$ & $\begin{array}{l}62.9 \\
60.7 \\
66.7 \\
50.0\end{array}$ & 0.57 & $>0.05$ \\
\hline $\begin{array}{l}\text { Clinical stage } \\
\text { high stage group (stage III-IV) } \\
\text { low stage group (stage I-II) }\end{array}$ & $\begin{array}{l}41 \\
27\end{array}$ & $\begin{array}{r}25 \\
2\end{array}$ & $\begin{array}{l}8 \\
9\end{array}$ & $\begin{array}{r}8 \\
16\end{array}$ & $\begin{array}{l}80.5 \\
40.7\end{array}$ & 6.73 & $<0.05$ \\
\hline $\begin{array}{l}\text { Lymph node metastasis } \\
\text { absent } \\
\text { present }\end{array}$ & $\begin{array}{l}20 \\
48\end{array}$ & $\begin{array}{r}2 \\
24\end{array}$ & $\begin{array}{r}5 \\
11\end{array}$ & $\begin{array}{l}13 \\
13\end{array}$ & $\begin{array}{l}35.0 \\
72.9\end{array}$ & 8.27 & $<0.05$ \\
\hline
\end{tabular}



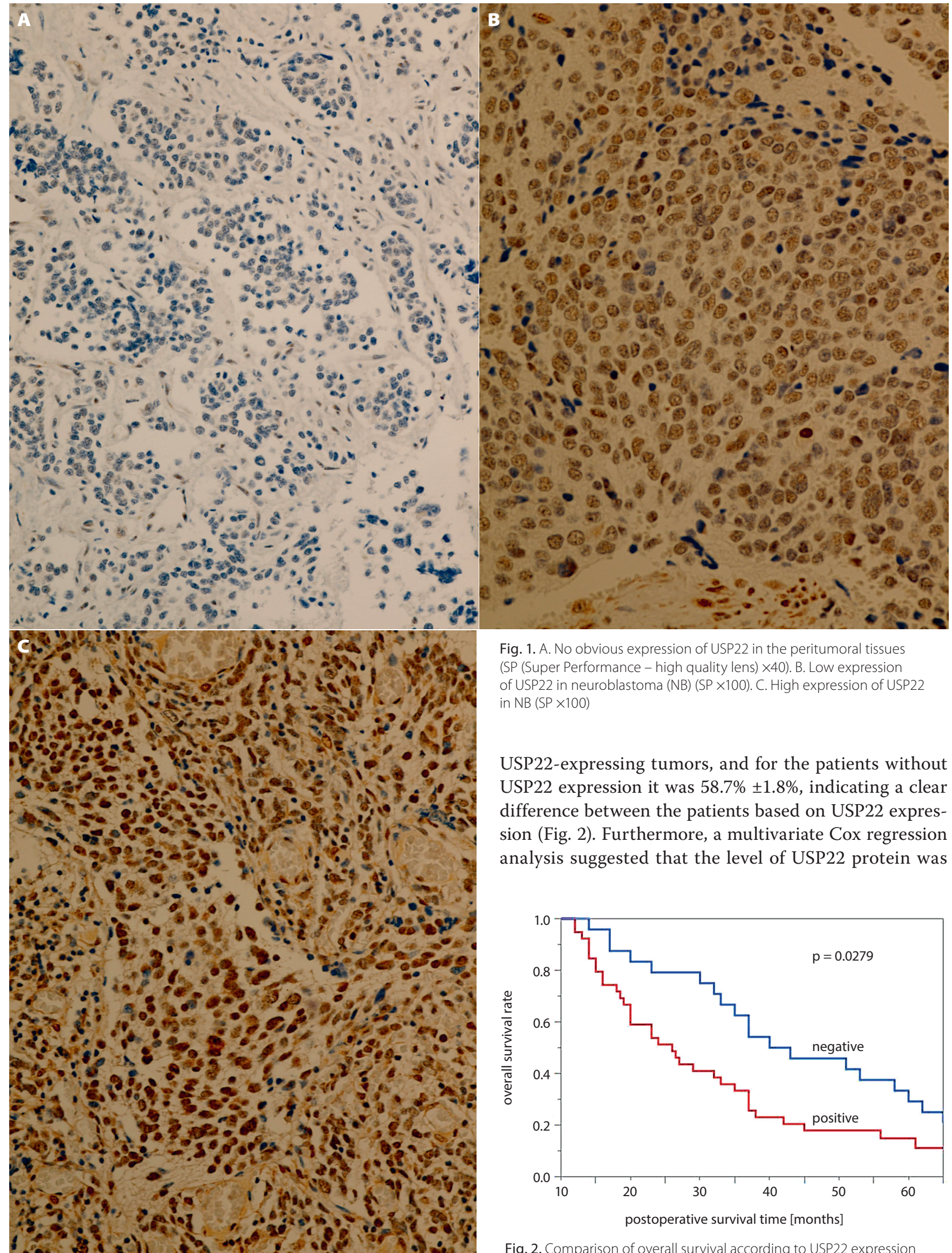

Fig. 1. A. No obvious expression of USP22 in the peritumoral tissues (SP (Super Performance - high quality lens) $\times 40$ ). B. Low expression of USP22 in neuroblastoma (NB) (SP $\times 100)$. C. High expression of USP22 in NB $(S P \times 100)$

USP22-expressing tumors, and for the patients without USP22 expression it was $58.7 \% \pm 1.8 \%$, indicating a clear difference between the patients based on USP22 expression (Fig. 2). Furthermore, a multivariate Cox regression analysis suggested that the level of USP22 protein was

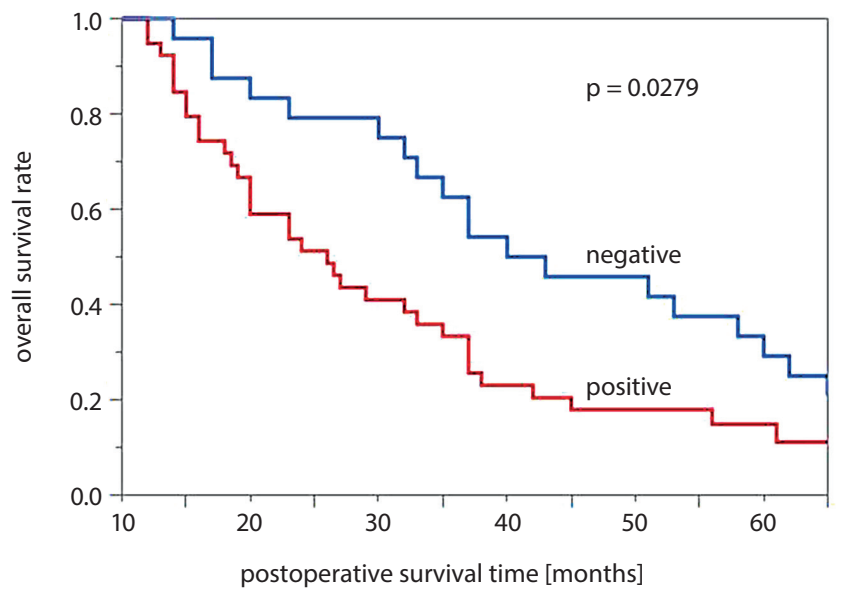

Fig. 2. Comparison of overall survival according to USP22 expression 
a predictive factor for survival $(\mathrm{p}<0.05)$. These findings demonstrated that detecting USP22 in patients with NB is a valuable biomarker for poor disease prognosis.

\section{Discussion}

One of the most common pediatric extracranial tumors is NB, which originates from the precursor cells of the sympathetic nervous system. The clinical presentation of NB varies greatly between patients, with some tumors undergoing spontaneous remission/regression, and others displaying malignant behavior. The rapid tumor progression, high degree of malignancy, metastasis and drug resistance, and its association with a poor prognosis are all factors which contribute to the challenges in treating NB. Although significant progress has been achieved in NB therapeutic strategies, 5-year event-free survival remains limited, with a rate of below $31 \%$ in children with high-risk NB. ${ }^{17}$ Thus, in determining new biomarkers for the development of personalized therapies, the elucidation of the mechanisms underlying metastasis and therapy resistance and the prediction of the disease prognosis are fundamental to improving the therapeutic outcomes of NB.

Although USP22 is considered a promising biomarker for NB diagnosis and prognosis, ${ }^{5-9,18-20}$ little is known about its effect in NB. Indeed, it is involved in the effective prediction of the response to therapy in individual patients with cancer. Furthermore, it is demonstrated to be centrally implicated in the pathogenesis of epithelial cancers and other solid tumors.

It is well-known that USP22, as part of hSAGA, deubiquitinates $\mathrm{H} 2 \mathrm{~A}$ and $\mathrm{H} 2 \mathrm{~B}$, thus editing the histone code. ${ }^{21}$ However, the mechanism by which USP22 is implicated in cancer progression and metastasis is unclear. Liu et al. ${ }^{21}$ demonstrated an oncogenic role of USP22 via the Polycomb complex protein BMI-1-mediated INK4a/ARF pathway and the protein kinase B (Akt) pathway. Furthermore, the alteration of the transcriptional regulator far upstream element binding protein 1 (FBP1) ubiquitination has also been proposed as a mechanism by which USP22 modulates tumorigenesis. ${ }^{22}$ Another critical mechanism contributing to the invasion and metastasis of various cancers is the epithelial-mesenchymal transition. ${ }^{23}$ Yang et al. demonstrated the implication of USP22 in spinocerebellar ataxia and its progression. ${ }^{24}$ Additional research is warranted to elucidate the connection between these USP22 functions and other chromatin-modifying systems in order to provide more insights into chromatin remodeling and USP22 gene activation.

To evaluate whether or not USP22 activation is implicated in NB initiation and/or progression, we acquired tissues from representative patients. Our results indicated a significantly increased expression of USP22 in the NB tissues compared with the peritumoral tissues, with approx. $61.76 \%$ of the NB samples tested positive for USP22.
Furthermore, our findings suggested a significant correlation between USP22 and clinicopathological parameters, including INSS stage and lymph node metastasis.

As evidenced by the Kaplan-Meier survival analysis, the patients with USP22-positive tumors displayed a worse overall survival rate than the patients with USP22-negative tumors. Thus, the increased expression of USP22 may be valuable in predicting poor prognosis, while suppressing USP22 may be a potential target for therapeutic intervention in NB. Furthermore - given that it might not just be the pure presence or absence of USP22 that is indicative - further, broader studies should examine the results as they correlate to the intensity or proportion of USP22 expression in the patients.

To our knowledge, this is the first study to indicate a specifically increased expression of USP22 in clinical NB samples and positive correlations between USP22 expression and NB progression and poor prognosis. These findings support USP22 as a potential candidate for identifying patients with NB with a poor prognosis and as a predictor of their therapeutic outcomes. However, this study had several limitations, including the small study population and short follow-up period. Therefore, further studies with a larger sample size and an extended follow-up period are still required in order to confirm presented observations.

\section{ORCID iDs}

Qu Zhibo (1) https://orcid.org/0000-0002-9591-9145

Liu Lianxin (D) https://orcid.org/0000-0001-9000-4344

\section{References}

1. Kiyonari S, Kadomatsu K. Neuroblastoma models for insights into tumorigenesis and new therapies. Expert Opin Drug Discov. 2015; 10(1):53-62.

2. Yang S, Zheng J, Xiao X, et al. SOX2 promotes tumorigenicity and inhibits the differentiation of I-type neuroblastoma cells. Int J Oncol. 2014;46(1):317-323.

3. Bansal D, Totadri S, Chinnaswamy G, et al. Management of neuroblastoma: ICMR Consensus Document. Indian J Pediatr. 2017;84(6): 446-455.

4. Glinsky GV. Death-from-cancer signatures and stem cell contribution to metastatic cancer. Cell Cycle. 2005;4(9):1171-1175.

5. Liu YL, Yang YM, Xu H, Dong XS. Increased expression of ubiquitinspecific protease 22 can promote cancer progression and predict therapy failure in human colorectal cancer. J Gastroenterol Hepatol. 2010;25(11):1800-1805.

6. He $\mathrm{Y}$, Jin $\mathrm{YJ}$, Zhang $\mathrm{YH}$, et al. Ubiquitin-specific peptidase 22 overexpression may promote cancer progression and poor prognosis in human gastric carcinoma. Transl Res. 2015;165(3):407-416.

7. Tang B, Liang X, Tang F, et al. Expression of USP22 and Survivin is an indicator of malignant behavior in hepatocellular carcinoma. Int J Oncol. 2015;47(6):2208-2216.

8. Hu J, Yang D, Zhang H, et al. USP22 promotes tumor progression and induces epithelial-mesenchymal transition in lung adenocarcinoma. Lung Cancer. 2015;88(3):239-245.

9. Zhang Y, Yao L, Zhang X, et al. Elevated expression of USP22 in correlation with poor prognosis in patients with invasive breast cancer. J Cancer Res Clin Oncol. 2011;137(8):1245-1253.

10. Melo-Cardenas J, Zhang Y, Zhang DD, Fang D. Ubiquitin-specific peptidase 22 functions and its involvement in disease. Oncotarget. 2016;7(28):44848-44856.

11. Ma Y, Fu HL, Wang Z, et al. USP22 maintains gastric cancer stem cell stemness and promotes gastric cancer progression by stabilizing BMI1 protein. Oncotarget. 2017;8(20):33329-33342. 
12. Vijayalingam S, Subramanian T, Zhao LJ, Chinnadurai G. The cellular protein complex associated with a transforming region of E1A contains c-MYC. J Virol. 2015;90(2):1070-1079.

13. Zhou D, Liu P, Sun DW, et al. USP22 down-regulation facilitates human retinoblastoma cell aging and apoptosis via inhibiting TERT/P53 pathway. Eur Rev Med Pharmacol Sci. 2017;21(12):2785-2792.

14. Ling S, Li J, Shan Q, et al. USP22 mediates the multidrug resistance of hepatocellular carcinoma via the SIRT1/AKT/MRP1 signaling pathway. Mol Oncol. 2017;11(6):682-695.

15. Zhang XY, Pfeiffer HK, Thorne AW, McMahon SB. USP22, an hSAGA subunit and potential cancer stem cell marker, reverses the polycomb-catalyzed ubiquitylation of histone H2A. Cell Cycle. 2008;7(11): 1522-1524.

16. Piao S, Liu Y, Hu J, et al. USP22 is useful as a novel molecular marker for predicting disease progression and patient prognosis of oral squamous cell carcinoma. PLoS One. 2012;7(8):e42540.

17. Maris JM. Recent advances in neuroblastoma. N Engl J Med. 2010; 362(23):2202-2211.

18. Liu YL, Yang YM, Xu H, Dong XS. Aberrant expression of USP22 is associated with liver metastasis and poor prognosis of colorectal cancer. J Surg Oncol. 2011;103(3):283-289.
19. Xiong J, Che X, Li X, Yu H, Gong Z, Li W. Cloning and characterization of the human USP22 gene promoter. PLoS One. 2012;7(12):e52716.

20. Wang L, Dent SY. Functions of SAGA in development and disease. Epigenomics. 2014;6(3):329-339.

21. Liu YL, Zheng J, Tang LJ, et al. The deubiquitinating enzyme activity of USP22 is necessary for regulating HeLa cell growth. Gene. 2015; 572(1):49-56.

22. Atanassov BS, Dent SY. USP22 regulates cell proliferation by deubiquitinating the transcriptional regulator FBP1. EMBO Rep. 2011;2(9): 924-930.

23. Li Y, Yang Y, Li J, et al. USP22 drives colorectal cancer invasion and metastasis via epithelial-mesenchymal transition by activating AP4. Oncotarget. 2017;8(20):32683-32695.

24. Yang H, Liu S, He WT, Zhao J, Jiang LL, Hu HY. Aggregation of polyglutamine-expanded ataxin 7 protein specifically sequesters ubiquitinspecific protease 22 and deteriorates its deubiquitinating function in the Spt-Ada-Gcn5-acetyltransferase (SAGA) complex. J Biol Chem. 2015;290(36):21996-22004. 